\title{
Community organizers
}

Electrochemical sensors monitor communication between bacterial aggregates.

For 'simple' organisms, bacteria can be remarkably democratic_coordinating their behavior on the basis of signals from immediate neighbors as well as from more distant aggregates. These quorum-sensing mechanisms tell individual bacteria when population density is high enough to begin activating virulence pathways or other functions.

Researchers at the University of Texas at Austin have combined their expertise in microbiology, chemistry and materials science to examine this process in remarkable detail. Marvin Whiteley's microbiology lab has been employing a technique developed by colleague Jason Shear for generating 3D-printed nanoscale protein 'cages', which allow them to monitor quorum sensing in small bacterial communities of well-defined size and scale. However, they found that conventional GFP-based reporters of quorum signaling proved inadequate. "GFP reporting isn't all that sensitive, particularly in prokaryotes," says Whiteley.

Now, Whiteley's group has employed a technique called scanning electrochemical microscopy (SECM), developed by collaborator Allen Bard. SECM detects electrochemically active molecules with high spatial and temporal resolution. The researchers focused on pyocyanin, a metabolite produced by the pathogenic bacterium Pseudomonas aeruginosa in response to quorum-sensing signals. After trapping cells within 3D-printed cages, they used SECM to scan for pyocyanin production as the bacteria proliferated. Remarkably, colonies of as few as 500 cells exhibited active quorum sensing - more than fivefold fewer than GFP-based estimates.

The researchers subsequently printed multiunit cages housing physically distinct $P$. aeruginosa communities separated by a porous, 8 -micrometer-thick wall. This setup revealed signaling between a colony of mutant bacteria that could send but not receive quorum signals and another colony for which the opposite was true. "To my knowledge, this is the first instance where people have confined bacterial populations at defined distances and asked if they can communicate," says Whiteley.

The flexibility of this approach should enable simulation of communication within a broad range of bacterial ecosystems. These studies will also be assisted by Bard's ongoing efforts to further refine SECM. "We're pushing hard to get our resolution down...so we can really learn things about individual bacteria," he says.

\section{Michael Eisenstein}

\section{RESEARCH PAPERS}

Connell, J.L. et al. Real-time monitoring of quorum sensing in 3D-printed bacterial aggregates using scanning electrochemical microscopy. Proc. Natl. Acad. Sci. USA 111, 18255-18260 (2014). 